\title{
Growth and Growth Velocity of Lean Body Mass and Fat in Adolescent Boys
}

\author{
JANA PAŘÍZKOVÁ \\ VU FTVS, Charles University, Prague, Czechoslovakia
}

\section{Extract}

Beginning at age 10 years, height, weight, body density, and bone age of $\mathbf{4 0}$ normal boys were measured yearly over a period of 8 years. From body density, lean body mass and body content of fat were calculated. Values for all measurements are included in the Appendix and mean values, including those for lean body mass and percentage of body fat, are presented for each year in Table 1 . Coefficients of correlation between measurements made during the first and eighth years of study were high for height $(0.68)$, weight $(0.50)$, and lean body mass $(0.60)$, but relatively low for body fat $(0.25)$. Correlation coefficients between the various parameters in a given year are presented in Table 2 .

The maximum yearly increment in height, weight, and lean body mass of individual subjects coincided in time. The respective mean values for the year of maximum growth were $9.4 \mathrm{~cm}, 8.1 \mathrm{~kg}$, and 7.5 kg. Throughout the growth spurt, the contribution of fat to increase in body weight was small. It may be concluded that, next to height, the absolute amount of lean body mass demonstrates the most constant trend of development during adolescence.

\section{Speculation}

Lean body mass, like weight and height, follows individual patterns that are consistent throughout adolescence. The trends in height and lean body mass are more constant than trends in body weight.

Incremental data for height and weight during childhood and adolescence (9) suggest that growing individuals generally adhere to a consistent pattern of growth, i.e., the increments observed over a period of years are reasonably predictive of the increments to be anticipated in the next few years unless illness, food deprivation, or other external events intervene.

The present report concerns yearly determinations over a period of 8 years of height, weight, body density, and bone age. The results permit calculation of incremental data for lean body mass.

\section{SUBJECTS AND METHODS}

One hundred forty-six males ranging from 10 years 2 months to 11 years 4 months were studied in 1961. Several reports concerning a majority of these subjects followed for less than the time reported here have already appeared (5-7). The present report concerns 40 subjects who were available for all yearly examinations over a period of 8 years. They are considered to be a representative subsample of the original cohort (8)

All subjects lived in Prague, a city of over 1 million inhabitants, under similar socioeconomic, cultural, and nutritional circum-

Table 1. Summary of yearly measurements of 40 boys 10 through 17 years of age

\begin{tabular}{|c|c|c|c|c|c|c|c|c|}
\hline \multirow[b]{2}{*}{ Measurements } & \multicolumn{8}{|c|}{ Year of observation } \\
\hline & 1 & 2 & 3 & 4 & 5 & 6 & 7 & 8 \\
\hline \multicolumn{9}{|l|}{ Age (years) } \\
\hline Mean & 10.7 & 11.7 & 12.7 & 13.7 & 14.6 & 15.7 & 16.6 & 17.7 \\
\hline $\mathrm{SD}$ & 0.40 & 0.45 & 0.33 & 0.32 & 0.34 & 0.37 & 0.69 & 0.36 \\
\hline \multicolumn{9}{|l|}{ Height $(\mathrm{cm})$} \\
\hline \multicolumn{9}{|l|}{ Weight (kg) } \\
\hline Mean & 36.0 & 39.4 & 44.0 & 50.0 & 56.8 & 62.8 & 66.6 & 69.2 \\
\hline $\mathrm{SD}$ & 4.09 & 4.50 & 5.72 & 7.44 & 8.27 & 7.56 & 6.40 & 6.28 \\
\hline \multicolumn{9}{|l|}{ Bone age (years) } \\
\hline Mean & 10.8 & 11.8 & 12.8 & 13.6 & 14.6 & 15.8 & 17.1 & 18.1 \\
\hline $\mathrm{SD}$ & 0.76 & 0.88 & 0.74 & 0.76 & 0.88 & 1.34 & 1.24 & 1.04 \\
\hline \multicolumn{9}{|l|}{ Body density } \\
\hline $\mathrm{SD}$ & 4.7 & 3.8 & 4.8 & 6.4 & 7.9 & 6.7 & 6.0 & 5.8 \\
\hline \multicolumn{9}{|l|}{ Fat (\% body wt) } \\
\hline Mean & 16.1 & 15.3 & 14.2 & 11.3 & 12.7 & 13.1 & 10.9 & 9.1 \\
\hline SD & 5.6 & 7.1 & 6.0 & 5.6 & 4.8 & 5.2 & 4.4 & 4.8 \\
\hline
\end{tabular}


Table 2. Selected correlation coefficients between height, weight, lean body mass $(L B M)$ and fat ${ }^{1}$ during each of 8 years of observation

\begin{tabular}{|c|c|c|c|c|c|c|c|c|}
\hline \multirow[b]{2}{*}{ Correlation coefficient } & \multicolumn{8}{|c|}{ Year of observation } \\
\hline & 1 & 2 & 3 & 4 & 5 & 6 & 7 & 8 \\
\hline Height/weight & 0.43 & 0.69 & 0.75 & 0.78 & 0.78 & 0.59 & $0.31^{2}$ & 0.41 \\
\hline Height/LBM & 0.54 & 0.75 & 0.81 & 0.89 & 0.84 & 0.66 & 0.58 & 0.59 \\
\hline Weight/LBM & 0.92 & 0.82 & 0.89 & 0.92 & 0.92 & 0.85 & 0.48 & 0.80 \\
\hline Weight/fat & 0.71 & $0.37^{2}$ & 0.51 & 0.43 & $0.27^{2}$ & 0.46 & $0.12^{2}$ & 0.45 \\
\hline
\end{tabular}

${ }^{1}$ Height expressed in centimeters and weight, LBM and fat in kilograms.

${ }^{2}$ Not significant; all other values significant $(p<0.05)$.

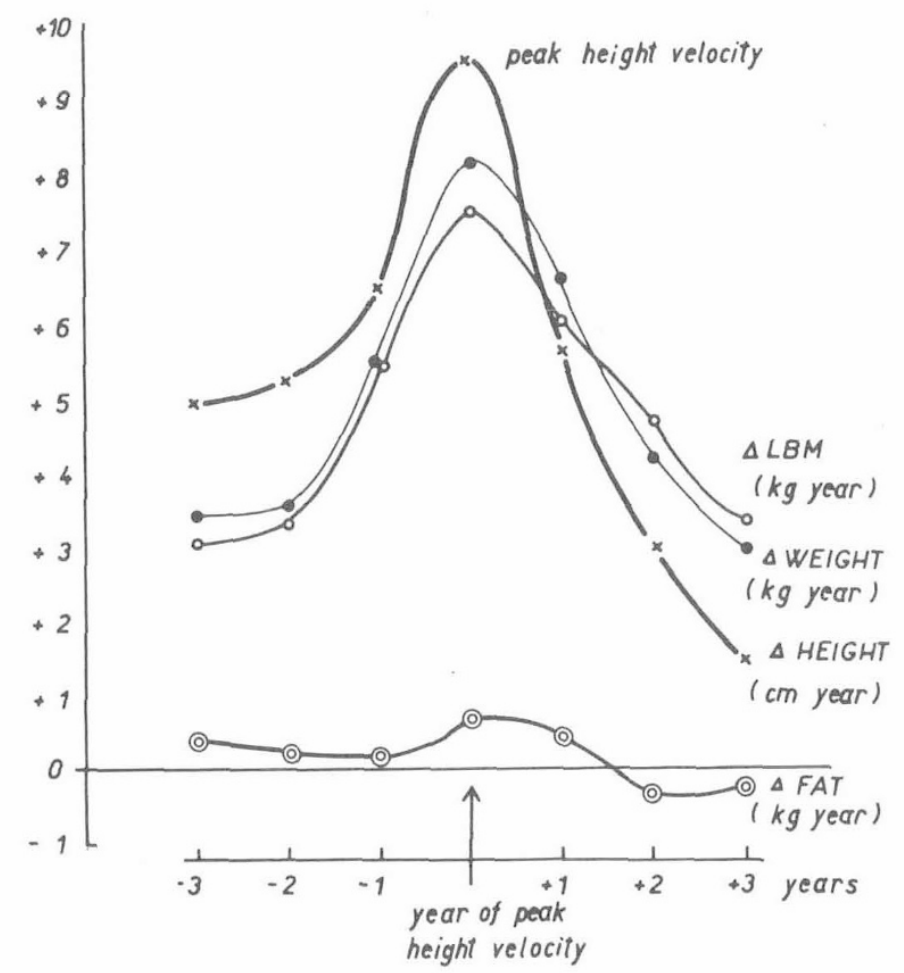

Fig. 1. Changes in body composition and weight according to peak height velocity in boys from 10.7 to 17.7 years $(\mathrm{n}=40)$. LBM: lean body mass.

stances. About one-fourth of the subjects were involved in vigorous athletic training, another one-fourth were relatively uninterested in sports or in athletic training but participated in compulsory physical training in school. The remaining one-half were intermediate in their physical activity.

Physical examination, anthropometric measurements, and determinations of body composition were made generally in the spring. Body density was measured $(3,4)$ by hydrostatic weighing and determination of residual lung volume by nitrogen dilution. Percentage of body fat was calculated according to the formula (3)

$$
\% \text { Body fat }=\left(\frac{4.201}{\text { density }}-3.813\right) \times 100
$$

Bone age was estimated from roentgenograms of the wrist (2). Reports of various physiologic measurements, including aerobic capacity and heart volume, made simultaneously, have been published elsewhere $(1,6)$. Participation in this study was voluntary and informed consent was obtained from subjects and parents.

\section{RESULTS}

Date of birth, date of each of the yearly measurements, and height, weight, bone age, and body density of each child are

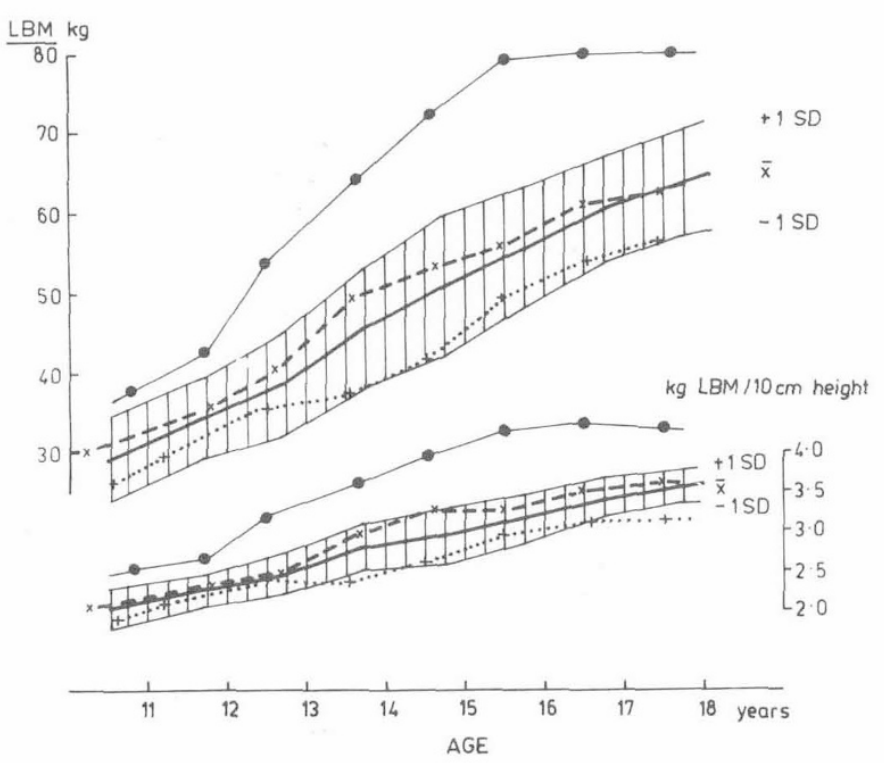

Fig. 2. Changes in lean body mass $(L B M)$ of three boys in relation to age during adolescence. The mean and \pm 1 SD for LBM of all 40 subjects is also indicated.

presented for each of the 8 years in the Appendix. Table 1 presents a summary of the measurements, including means and standard deviations for age, height, weight, bone age, body density, lean body mass, and percentage of body weight accounted for by fat. Bone age corresponded rather closely to chronologic age.

Increases in height, weight, and lean body mass were most rapid between the third and sixth years of observation (mean ages 12.2 to 15.7 years). Yearly gains in height, weight, and lean body mass during this period averaged $6.7 \mathrm{~cm}, 6.5 \mathrm{~kg}$, and $5.8 \mathrm{~kg}$, respectively.

Coefficients of correlation between measurements made during the first and the eighth year of study were 0.68 for height, 0.50 for weight, 0.60 for lean body mass (in kilograms) and 0.25 for fat (in kilograms). These correlation coefficients were statistically significant $(p<0.01$ for height, weight, and lean body mass and $p<$ 0.05 for fat). When lean body mass and fat were expressed as percentages of body weight, the correlation coefficients were reduced to 0.20 and 0.20 , respectively; these correlations were not statistically significant. Correlations between the various parameters at a given year are presented in Table 2.

To gain insight into the magnitude and nature of changes in body composition during the adolescent growth spurt, incremental data have been assembled in Figure 1 in relation to the year in which peak height velocity was attained. The maximal yearly increments in height, weight, and lean body mass were $9.4 \mathrm{~cm}, 8.1$ $\mathrm{kg}$, and $7.5 \mathrm{~kg}$, respectively, the maximum increment in each parameter occurring during the same year. The contribution of fat to increase in body weight was small. 
Figure 2 presents data on lean body mass considered in relation to age. The three individuals, differing in the absolute magnitude of the yearly increments, maintained their relative position through the years. The majority of the subjects followed a consistent pattern of increases in lean body mass demonstrated. The consistency of the individual patterns was not improved when lean body mass was related to height (lower panel of Fig. 2)-a modification of Quetelet's index characterizing body build.

\section{COMMENT AND SUMMARY}

The incremental data based on longitudinal observations made over a period of 8 years provide valuable information about physical growth of boys during adolescence. Of considerable interest is the observation that the peak increment in height coincides with the peak increment in weight and in lean body mass. The presence of individual consistency of body proportions during adolescent growth has been known from longitudinal measurements of weight and height. The present study presents evidence that lean body mass also follows individual patterns that are consistent throughout adolescence. The trends in height and lean body mass appear to be more constant than the trends in body weight.

\section{REFERENCES AND NOTES}

1. Čermák, J.: Die Änderungen des Herzvolumens in der Entwicklungsperiode bei 12-15 jährigen Knaben im Vergleich mit den Veränderungen der somatometrischen Grundkriterien. Cardiologia (Basel), 53: 99 (1968).

2. Greulich, W. W., and Pyle, S. I.: Radiographic Atlas of Skeletal Development of the Hand and Wrist, Ed. 2 (Stanford University Press, Stanford, 1959).

3. Keys, A., and Brožek, J.: Body fat in adult man. Physiol. Rev., 33: 245 (1953).

4. Parízková, J.: Age trends in fat in normal and obese children. J. Appl. Physiol., 16: $173(1961)$.

5. Paŕízková, J.: Longitudinal study of the development of body composition and body build in boys of various physical activity. Human Biol., 40: 212 (1968).

6. Paŕízková, J.: Interrelationships between body size, body composition and function. In: H. Roche and F. Falkner: Nutrition and Malnutrition. Advances in Experimental Medicine and Biology, Vol. 49, p. 119 (Plenum Press, New York, 1974)

7. Parízková, J., and Carter, J. E. L.: Influence of physical activity on stability of somatotypes in boys. Amer. J. Phys. Anthropol. (In press.)

8. Šprynarová, Š.: Longitudinal study of the influence of different physical activity programs on functional capacity of the boys from 11 to 18 years. Acta Paediat. Belg. 28: 204 (1974).

9. Tanner, J. M., Whitehouse, R. H., and Takaishi, M.: Standards from birth to maturity for height, weight, height velocity, and weight velocity: British children, 1965. Arch. Dis. Childhood, 41: 454 (1966); Part II, 41: 613 (1966).

10. Requests for reprints should be addressed to: J. Pařizková, VÚ FTVS, Charles University, Prague (Czechoslovakia); or to: S. J. Fomon, Department of Pediatrics, College of Medicine, Iowa City, Iowa, 52242 (USA).

11. Accepted for publication January 28, 1976.

\section{APPENDIX}

Appendix. Date of birth, date of measurement, height, weight, bone age and body density of individual subjects.

\begin{tabular}{|c|c|c|c|c|c|c|c|c|c|c|c|c|c|c|c|c|c|c|c|c|c|}
\hline \multicolumn{7}{|c|}{1961} & \multicolumn{5}{|c|}{1962} & \multicolumn{5}{|c|}{1963} & \multicolumn{5}{|c|}{1964} \\
\hline $\begin{array}{l}\text { Subject } \\
\text { Number }\end{array}$ & $\begin{array}{l}\text { Date of } \\
\text { Birth }\end{array}$ & $\begin{array}{l}\text { Date of } \\
\text { measure- } \\
\text { ment }\end{array}$ & $\begin{array}{l}\text { He ight, } \\
\mathrm{cm}\end{array}$ & $\begin{array}{c}\text { Weight, } \\
\mathrm{kg}\end{array}$ & $\begin{array}{l}\text { Bone } \\
\text { age, } \\
\text { years }\end{array}$ & $\begin{array}{c}\text { Density, } \\
g / m 1\end{array}$ & $\begin{array}{l}\text { Date of } \\
\text { measure- } \\
\text { ment }\end{array}$ & $\begin{array}{l}\text { Height, } \\
\mathrm{cm}\end{array}$ & $\begin{array}{c}\text { Height, } \\
\mathrm{kg}\end{array}$ & $\begin{array}{l}\text { Bone } \\
\text { age, } \\
\text { years }\end{array}$ & $\underset{g / m 1}{\text { Density, }}$ & $\begin{array}{l}\text { Date of } \\
\text { measure- } \\
\text { ment }\end{array}$ & $\underset{\mathrm{cm}}{\text { Height, }}$ & $\begin{array}{l}\text { Weight, } \\
\mathrm{kg}\end{array}$ & $\begin{array}{l}\text { Bone } \\
\text { age, } \\
\text { years }\end{array}$ & $\begin{array}{l}\text { Density, } \\
\mathrm{g} / \mathrm{ml}\end{array}$ & $\begin{array}{l}\text { Date of } \\
\text { measure- } \\
\text { ment }\end{array}$ & $\underset{\mathrm{Chi}}{\mathrm{H}} \mathrm{Height,}$ & $\underset{\mathrm{kg}}{\text { Height, }}$ & $\begin{array}{l}\text { Bone } \\
\text { age, } \\
\text { years }\end{array}$ & $\begin{array}{l}\text { Density. } \\
g / m 1\end{array}$ \\
\hline 1. & $2 / 13 / 51$ & $4 / 25$ & 144.5 & 33.30 & 10.5 & 1.078 & $2 / 26$ & 148.5 & 36.35 & 11.5 & 1.076 & $4 / 29$ & 154.0 & 40.12 & 12.5 & 1.084 & $5 / 14$ & 159.5 & 42.71 & 12,75 & \\
\hline 2. & $8 / 18 / 50$ & $\begin{array}{l}4 / 23 \\
6 / 01\end{array}$ & $\begin{array}{l}144.3 \\
147.5\end{array}$ & 35.84 & 11.5 & 1.056 & $\begin{array}{l}6 / 05 \\
6 / 05\end{array}$ & 153.5 & 39.36 & 12.0 & 1.100 & $5 / 14$ & 163.2 & 46.32 & 13.25 & 1.076 & $\begin{array}{l}4 / 16 \\
4 / 14\end{array}$ & 172.3 & $\begin{array}{l}42.71 \\
55.78\end{array}$ & 14.5 & $\begin{array}{l}1.080 \\
1.084\end{array}$ \\
\hline 3. & $1 / 12 / 51$ & $6 / 12$ & 147.0 & $\begin{array}{l}30.00 \\
40.00\end{array}$ & 10.5 & 1.054 & $5 / 15$ & 152.0 & 41.46 & 11.0 & 1.064 & $6 / 06$ & 158.5 & 49.21 & 12.75 & 1.053 & $5 / 26$ & 167.8 & 53.33 & 14.25 & 1.078 \\
\hline 4. & $12 / 25 / 50$ & $4 / 15$ & 140.5 & 29.32 & 9.5 & 1.059 & $4 / 17$ & 145.5 & 32.04 & 10.0 & 1.055 & $4 / 02$ & 149.2 & 36.92 & 11.0 & 1.056 & $5 / 26$ & $156.4^{\circ}$ & 42.48 & 12.0 & 1.063 \\
\hline 5. & $2 / 18 / 50$ & $6 / 01$ & 148.3 & 36.66 & 11.25 & 1.048 & $4 / 26$ & 152.8 & 39.65 & 12.5 & 1.048 & $5 / 06$ & 160.3 & 46.02 & 13.5 & 1.086 & $5 / 11$ & 169.5 & 53.22 & 14.75 & 1.077 \\
\hline 6. & $6 / 18 / 50$ & $5 / 04$ & 143.0 & 34.69 & 10.0 & 1074 & $4 / 10$ & 148.0 & 38.20 & 11.0 & 1.069 & $9 / 26$ & 156.4 & 44.25 & 12.25 & 1.072 & $6 / 23$ & 162.2 & 47.65 & 13.0 & 1.075 \\
\hline 7. & $2 / 13 / 51$ & $6 / 19$ & 150.2 & 46.45 & 10.5 & 1.037 & $4 / 28$ & 154.5 & 47.64 & 11.5 & 1.036 & $4 / 11$ & 159.0 & 52.13 & 12.5 & 1.050 & $6 / 09$ & 166.4 & 58.25 & 13.5 & 1.053 \\
\hline 8. & $9 / 14 / 50$ & $5 / 15$ & 151.3 & 41.40 & 12.0 & 1.078 & $4 / 10$ & 158.0 & 46.50 & 13.0 & 1.077 & $4 / 04$ & 168.7 & 57.40 & 14.0 & 1.085 & $5 / 11$ & 178.0 & 69.78 & 15.5 & 1.079 \\
\hline 9. & $7 / 10 / 50$ & $4 / 17$ & 133.5 & 29.51 & 9.5 & 1.045 & $2 / 26$ & 137.2 & 33.34 & 10.25 & 1.072 & $4 / 13$ & & & 12.0 & & $5 / 21$ & 153.0 & 41.66 & 13.0 & 1.078 \\
\hline 10. & $5 / 09 / 50$ & $4 / 21$ & 140.0 & 38.58 & 10.0 & & $6 / 20$ & 147.5 & 43.90 & 11.0 & 1.044 & $4 / 22$ & 153.0 & 50.35 & 13.0 & 1.054 & $4 / 20$ & 163.4 & 58.02 & 14.0 & 1.056 \\
\hline i1. & $5 / 111 / 50$ & 6/05 & 142.3 & 42.40 & 10.75 & $\begin{array}{l}1.047 \\
1.061\end{array}$ & $5 / 14$ & 147.5 & 46.90 & 11.5 & 1.031 & $4 / 27$ & 153.0 & 52.73 & 12.5 & 1.028 & $4 / 27$ & 161.4 & 58.00 & 13.5 & 1.056 \\
\hline 12. & $10 / 16 / 50$ & $5 / 04$ & 137.0 & 32.02 & & $\begin{array}{l}1.061 \\
1.074\end{array}$ & $2 / 27$ & 142.0 & 34.83 & 13.0 & 1.072 & $3 / 26$ & 146.5 & 36.43 & 13.5 & 1.071 & $5 / 11$ & 150.2 & 40.53 & 14.0 & 1.071 \\
\hline 13. & $1 / 13 / 50$ & $5 / 18$ & 156.3 & 38.50 & $\begin{array}{l}12.0 \\
10.5\end{array}$ & $\begin{array}{l}1.074 \\
1.049\end{array}$ & $\begin{array}{l}6 / 13 \\
4 / 17\end{array}$ & 163.2 & 41.80 & 12.5 & 1.084 & $4 / 25$ & 169.3 & 45.85 & 13.5 & 1.071 & $4 / 07$ & 177.3 & 55.20 & 14.25 & 1.081 \\
\hline 14. & $11 / 05 / 50$ & $5 / 23$ & 135.5 & $\begin{array}{l}28.90 \\
40.30\end{array}$ & $\begin{array}{l}10.5 \\
12.0\end{array}$ & $\begin{array}{l}1.049 \\
1.065\end{array}$ & $\begin{array}{l}4 / 17 \\
6 / 12\end{array}$ & 145.2 & 31.70 & 11.5 & 1.036 & $6 / 04$ & & 34.37 & 12.5 & 1.077 & $10 / 21$ & 158.2 & 39.75 & 13.0 & 1.063 \\
\hline 15. & $3 / 25 / 51$ & $5 / 30$ & 149.8 & $\begin{array}{l}40.30 \\
37.75\end{array}$ & $\begin{array}{l}12.0 \\
11.25\end{array}$ & $\begin{array}{l}1.065 \\
1.042\end{array}$ & $6 / 12$ & 154.5 & 43.16 & 13.5 & 1.051 & $5 / 21$ & 159.7 & 46.10 & 14.0 & 1.052 & $6 / 11$ & 165.2 & 49.70 & 14.25 & 1.062 \\
\hline 16. & $6 / 21 / 50$ & $5 / 25$ & 149.0 & $\begin{array}{l}37.75 \\
33.10\end{array}$ & $\begin{array}{l}111.25 \\
11.0\end{array}$ & $\begin{array}{l}1.042 \\
1.045\end{array}$ & $\begin{array}{l}6 / 13 \\
5 / 31\end{array}$ & $\begin{array}{l}154.8 \\
144.2\end{array}$ & 41.85 & 12.5 & 1.071 & $4 / 09$ & 160.6 & 46.25 & 13.0 & 1.064 & $4 / 14$ & 170.0 & 54.50 & 14.25 & 1.074 \\
\hline 17. & $8 / 20 / 50$ & $4 / 25$ & 139.5 & $\begin{array}{l}33.10 \\
30.40\end{array}$ & $\begin{aligned} 11.0 \\
9.5\end{aligned}$ & $\begin{array}{l}1.045 \\
1.065\end{array}$ & $\begin{array}{l}5 / 31 \\
4 / 17\end{array}$ & 144.2 & 35.80 & 11.5 & 1.066 & $4 / 18$ & 148.2 & 37.90 & 12.5 & & $6 / 08$ & 155.8 & 42.95 & 12.75 & 1.076 \\
\hline 18. & $10 / 25 / 50$ & $4 / 21$ & 136.3 & $\begin{array}{l}30.40 \\
30.83\end{array}$ & $\begin{array}{l}9.5 \\
9.5\end{array}$ & 1.034 & $11 / 06$ & $\begin{array}{l}140.0 \\
148.0\end{array}$ & $\begin{array}{l}32.50 \\
35\end{array}$ & 10.25 & 1.078 & $5 / 16$ & 145.1 & 35.60 & 11.0 & 1.100 & $6 / 22$ & 151.5 & 38.83 & 12.0 & 1.078 \\
\hline 19. & $11 / 18 / 50$ & $5 / 29$ & 141.0 & $\begin{array}{l}30.83 \\
33.30\end{array}$ & $\begin{array}{r}9.5 \\
11.0\end{array}$ & $\begin{array}{l}1.034 \\
1.060\end{array}$ & $6 / 06$ & $\begin{array}{l}148.0 \\
149.5\end{array}$ & $\begin{array}{l}35.02 \\
36.66\end{array}$ & 11.5 & 1.100 & $4 / 22$ & 150.1 & 35.80 & 12. & 1.0 & $6 / 29$ & 160.0 & 43.40 & 13.5 & \\
\hline 20. & $6 / 21 / 50$ & $5 / 26$ & 144.5 & $\begin{array}{l}33.30 \\
33.40\end{array}$ & $\begin{array}{l}11.0 \\
11.0\end{array}$ & $\begin{array}{l}1.060 \\
1.057\end{array}$ & $5 / 11$ & $\begin{array}{r}149.5 \\
48.0\end{array}$ & $\begin{array}{r}36.66 \\
38.00\end{array}$ & 12.0 & 1.054 & $4 / 27$ & 154.0 & 40.35 & 12.75 & & $6 / 01$ & 150.3 & 44.35 & 13.5 & \\
\hline 21. & $10 / 28 / 50$ & $11 / 13$ & 144.5 & $\begin{array}{l}37.40 \\
34.28\end{array}$ & 11.0 & 1.061 & $4 / 10$ & $\begin{array}{l}48.0 \\
148.4\end{array}$ & $\begin{array}{r}38.00 \\
37.50\end{array}$ & 12.0 & 1.047 & $4 / 25$ & 153.1 & 42.00 & 12.75 & 1.056 & $5 / 19$ & 165.1 & 49.76 & 13.5 & 1.072 \\
\hline 22. & $2 / 06 / 51$ & $5 / 30$ & 145.0 & $\begin{array}{r}34.28 \\
38.31\end{array}$ & $\begin{array}{l}10.25 \\
12.0\end{array}$ & 1.085 & $4 / 19$ & 150.7 & $\begin{array}{l}37.50 \\
43.30\end{array}$ & 11.0 & 1.046 & $4 / 04$ & 156.0 & 42.85 & 12.0 & 1.055 & $5 / 25$ & 166.0 & 52.06 & 13.0 & 1.074 \\
\hline 23. & $4 / 22 / 50$ & $6 / 12$ & 144.8 & $\begin{array}{l}38.31 \\
38.80\end{array}$ & $\begin{array}{l}12.0 \\
10.75\end{array}$ & 1.039 & $4 / 24$ & 153.0 & $\begin{array}{l}43.30 \\
39.90\end{array}$ & 12.5 & 1.080 & 3/28 & 158.0 & 50.00 & 13.5 & 1.073 & $4 / 07$ & & 60.35 & & \\
\hline 24. & $8 / 26 / 50$ & $4 / 28$ & 148.5 & $\begin{array}{l}38.80 \\
36.53\end{array}$ & $\begin{array}{l}10.75 \\
11.5\end{array}$ & 1.049 & $6 / 08$ & 150.2 & $\begin{array}{l}39.90 \\
40.00\end{array}$ & $\begin{array}{l}12.0 \\
12.0\end{array}$ & 1.047 & $5 / 13$ & 158.5 & 45.21 & 13.0 & 1.071 & $4 / 20$ & 169.0 & 53.00 & 14.5 & 1.088 \\
\hline 25. & $9 / 11150$ & $5 / 25$ & 145.0 & $\begin{array}{l}36.53 \\
31.50\end{array}$ & 11.0 & 1.084 & $5 / 24$ & 142.5 & $\begin{array}{l}40.00 \\
33.70\end{array}$ & $\begin{array}{l}12.0 \\
12.5\end{array}$ & 1.052 & $4 / 23$ & 154.5 & 42.30 & 13.0 & 1.0 & $4 / 21$ & 162.2 & 47.32 & 13.75 & 1.075 \\
\hline 26. & $\begin{array}{r}10 / 23 / 50 \\
6 / 02 / 50\end{array}$ & $6 / 21$ & 136.8 & $\begin{array}{l}31.50 \\
34.65\end{array}$ & 111.5 & 1.068 & $5 / 31$ & 151.3 & $\begin{array}{l}33.70 \\
37.63\end{array}$ & & 1.088 & $6 / 06$ & 145.0 & 36.30 & 13.0 & 1.073 & $6 / 04$ & 149.1 & 39.74 & 13.5 & 1.075 \\
\hline 27. & $\begin{array}{l}6 / 02 \\
8 / 09\end{array}$ & $\begin{array}{l}6 / 09 \\
5 / 22\end{array}$ & 146.2 & $\begin{array}{l}34.65 \\
34.92\end{array}$ & & 1.065 & $6 / 04$ & 151.0 & 38.23 & $\begin{array}{l}12.5 \\
12.5\end{array}$ & 1.068 & $4 / 09$ & 156.5 & 41.63 & 13.5 & 1.0 & $6 / 02$ & 165.1 & 48.70 & 14.25 & 1.082 \\
\hline 28. & $\begin{array}{r}8 / 09 / 50 \\
10 / 25 / 50\end{array}$ & $\begin{array}{l}5 / 22 \\
6 / 23\end{array}$ & 145.0 & & 10.5 & 1.063 & $6 / 19$ & 152.1 & 39.30 & $\begin{array}{l}12.5 \\
12.5\end{array}$ & 1.067 & $4 / 27$ & 156.5 & 43.00 & 13.0 & 1.076 & $10 / 29$ & 171.0 & 54.20 & 14.0 & 1.088 \\
\hline 29. & $\begin{array}{r}10 / 25 / 50 \\
3 / 20 / 50\end{array}$ & $\begin{array}{r}6 / 23 \\
11 / 20\end{array}$ & 143.5 & $\begin{array}{l}34.94 \\
38.76\end{array}$ & 10.75 & 1.058 & $4 / 09$ & 149.0 & 40.74 & $\begin{array}{l}12.5 \\
11.0\end{array}$ & & $6 / 03$ & 162.0 & 44.60 & 14.0 & 1.072 & $5 / 11$ & 168.5 & 50.77 & 14.75 & 1.095 \\
\hline 30. & & $\begin{array}{r}11 / 20 \\
608\end{array}$ & 148.0 & $\begin{array}{l}38.76 \\
40.91\end{array}$ & 11.5 & 1.042 & $5 / 05$ & 151.0 & 46.80 & 12.75 & $\begin{array}{l}1.019 \\
1.033\end{array}$ & $3 / 25$ & 152.5 & 41.60 & 12.0 & 1.042 & $5 / 28$ & 158.2 & 41.10 & 12.75 & 1.057 \\
\hline 31. & $\begin{array}{l}1 / 21 / 50 \\
5 / 23 / 50\end{array}$ & $\begin{array}{l}6 / 08 \\
5112\end{array}$ & $\begin{array}{l}147.0 \\
148.6\end{array}$ & $\begin{array}{l}40.91 \\
36.26\end{array}$ & 11.25 & 1.069 & $6 / 25$ & 154.6 & 37.20 & 12.25 & & $5 / 07$ & 155.6 & 52.10 & 13.5 & 1.034 & $5 / 18$ & 162.0 & 59.93 & 14.0 & 1.033 \\
\hline 32. & $\begin{array}{l}5 / 23 / 50 \\
6 / 13 / 50\end{array}$ & $\begin{array}{l}5 / 12 \\
4 / 17\end{array}$ & $\begin{array}{l}148.6 \\
148.3\end{array}$ & $\begin{array}{l}36.26 \\
35.90\end{array}$ & 11.0 & 1.060 & $11 / 05$ & 156.3 & 44.40 & 13.0 & $\begin{array}{l}1.075 \\
1.046\end{array}$ & $6 / 03$ & 159.5 & 42.39 & 12.75 & 1.063 & $4 / 20$ & 165.5 & 47.75 & 13.5 & 1.079 \\
\hline 33. & & $6 / 22$ & 141.0 & 31.40 & 10.0 & 1.068 & $5 / 15$ & 145.3 & 33.63 & 10.5 & 1.076 & & 159.0 & $\begin{array}{l}45.75 \\
36.78\end{array}$ & 13.5 & 1.049 & $4 / 21$ & 163.2 & 49.70 & 14.25 & 1.056 \\
\hline 35. & $7 / 20 / 50$ & $5 / 23$ & $\begin{array}{l}14.0 \\
135.0\end{array}$ & 39.32 & 9.0 & 1.039 & $4 /$ & 139.8 & 44.40 & 10.5 & 1.043 & $\begin{array}{l}5 / 28 \\
4 / 111\end{array}$ & $\begin{array}{l}150.3 \\
148.0\end{array}$ & $\begin{array}{l}36.78 \\
55.60\end{array}$ & 11.0 & 1.049 & $5 / 05$ & 156.3 & 41.32 & 12.5 & 1.063 \\
\hline 36. & $11 / 11 / 50$ & $6 / 12$ & 133.5 & 32.19 & 10.0 & 1.056 & $5 / 17$ & 137.5 & 35.90 & 11.0 & 1.049 & $5 / 16$ & $\begin{array}{l}488.0 \\
142.0\end{array}$ & $\begin{array}{l}51.60 \\
37.12\end{array}$ & 12.75 & 1.057 & $6 / 11$ & 157.2 & 58.94 & 14.0 & 1.061 \\
\hline 37. & $3 / 17 / 51$ & $5 / 15$ & $\begin{array}{l}13.5 \\
143.0\end{array}$ & 38.30 & 10.25 & 1.055 & $4 / 28$ & 147.0 & 41.15 & 11.0 & 1.0 & $\begin{array}{l}5 / 16 \\
3 / 26\end{array}$ & $\begin{array}{l}142.0 \\
153.2\end{array}$ & $\begin{array}{l}37.12 \\
46.15\end{array}$ & 12.0 & 1.075 & $5 / 07$ & 146.0 & 40.80 & 12.75 & 1.055 \\
\hline 38. & $1 / 19 / 51$ & $6 / 01$ & $\begin{array}{l}14.0 \\
146.0\end{array}$ & 43.41 & 10.5 & 1.027 & $6 / 05$ & 151.9 & 48.75 & 11.5 & 1.0 & $4 / 28$ & 156.2 & $\begin{array}{l}46.15 \\
52.60\end{array}$ & $\begin{array}{l}12.0 \\
12.5\end{array}$ & $\begin{array}{l}1.040 \\
1.043\end{array}$ & $6 / 109$ & 163.1 & 54.15 & 13.0 & 1.058 \\
\hline 39. & $4 / 21 / 50$ & $5 / 02$ & 149.5 & 33.30 & 11.0 & 1.056 & $5 / 15$ & 155.5 & 36.00 & 12.5 & 1.050 & $5 / 14$ & 162.0 & $\begin{array}{l}52.60 \\
41.28\end{array}$ & $\begin{array}{l}12.5 \\
130\end{array}$ & $\begin{array}{l}1.043 \\
1.074\end{array}$ & $\begin{array}{l}4 / 14 \\
5 / 03\end{array}$ & 161.4 & 63.00 & 13.5 & 1.024 \\
\hline 40. & $2 / 03 / 50$ & $5 / 05$ & 142.6 & 35.22 & 11.25 & 1.062 & $5 / 25$ & 148.5 & 40.60 & 12.5 & 1.057 & $4 / 09$ & 152.3 & $\begin{array}{l}43.20 \\
43.20\end{array}$ & $\begin{array}{l}13.0 \\
13.25\end{array}$ & $\begin{array}{l}1.074 \\
1.046\end{array}$ & $\begin{array}{l}5 / 03 \\
5 / 28\end{array}$ & $\begin{array}{l}171.5 \\
160.5\end{array}$ & $\begin{array}{l}49.90 \\
49.50\end{array}$ & $\begin{array}{l}13.5 \\
13.75\end{array}$ & $\begin{array}{l}1.084 \\
1.063\end{array}$ \\
\hline
\end{tabular}




\begin{tabular}{|c|c|c|c|c|c|c|c|c|c|c|c|c|c|c|c|c|c|c|c|c|c|}
\hline \multicolumn{7}{|c|}{1965} & \multicolumn{5}{|c|}{1956} & \multicolumn{5}{|c|}{1967} & \multicolumn{5}{|c|}{1953} \\
\hline $\begin{array}{l}\text { Subject } \\
\text { Number }\end{array}$ & $\begin{array}{l}\text { Date of } \\
\text { Birth }\end{array}$ & $\begin{array}{l}\text { Date of } \\
\text { measure- } \\
\text { ment }\end{array}$ & $\underset{\mathrm{cm}}{\text { Height, }}$ & $\begin{array}{c}\text { Weight, } \\
\mathrm{kg}\end{array}$ & $\begin{array}{l}\text { Bone } \\
\text { age, } \\
\text { years }\end{array}$ & $\begin{array}{l}\text { Density, } \\
g / m 1\end{array}$ & $\begin{array}{l}\text { Date of } \\
\text { measure- } \\
\text { ment }\end{array}$ & $\begin{array}{l}\text { Height, } \\
\mathrm{cm}\end{array}$ & $\begin{array}{l}\text { Weight, } \\
\mathrm{kg}\end{array}$ & $\begin{array}{l}\text { Bone } \\
\text { age, } \\
\text { years }\end{array}$ & $\begin{array}{l}\text { Derisity, } \\
\mathrm{g} / \mathrm{ml}\end{array}$ & $\begin{array}{l}\text { Date of } \\
\text { measure- } \\
\text { ment }\end{array}$ & $\begin{array}{c}\text { Height, } \\
\mathrm{cm}\end{array}$ & $\begin{array}{c}\text { Weight, } \\
\mathrm{kg}\end{array}$ & $\begin{array}{l}\text { Bone } \\
\text { age, } \\
\text { years }\end{array}$ & $\begin{array}{l}\text { Density, } \\
\mathrm{g} / \mathrm{ml}\end{array}$ & $\begin{array}{l}\text { Date of } \\
\text { measure- } \\
\text { ment }\end{array}$ & $\begin{array}{c}\text { Height, } \\
\mathrm{cm}\end{array}$ & , Weight, & $\begin{array}{l}\text { Bone } \\
\text { age, } \\
\text { years }\end{array}$ & $\begin{array}{l}\text { Density, } \\
\text { g/mI }\end{array}$ \\
\hline 1. & $2 / 13 /$ & $4 / 06$ & 165.0 & 45.50 & 73.5 & 1.086 & $4 / 21$ & 175.0 & 57.50 & 14.0 & 1.063 & $5 / 06$ & 182.8 & 63.10 & 15.0 & 1.082 & $4 / 29$ & 185.0 & & 15.75 & 1.082 \\
\hline 2. & $8 / 18 / 50$ & $4 / 28$ & 178.1 & 65.15 & $\begin{array}{l}15.5 \\
15.25\end{array}$ & 1.082 & $5 / 25$ & 180.5 & 69.40 & 17.25 & 1.065 & $4 / 18$ & 181.5 & 71.80 & 18 & 1. 070 & $4 / 29$ & 181.6 & 74.20 & 19.0 & $\begin{array}{l}1.082 \\
1.095\end{array}$ \\
\hline 3. & $\begin{array}{r}1 / 12 / 51 \\
12 / 25 / 50\end{array}$ & $\begin{array}{l}4 / 07 \\
3 / 15\end{array}$ & $\begin{array}{l}175.0 \\
161.0\end{array}$ & $\begin{array}{l}59.40 \\
46.00\end{array}$ & $\begin{array}{l}15.25 \\
13.0\end{array}$ & $\begin{array}{l}1.080 \\
1.073\end{array}$ & $5 / 17$ & 180.0 & 61.90 & 16.75 & 1.090 & $5 / 15$ & 182.0 & 68.40 & 17.75 & 1.079 & $4 / 29$ & 184.5 & 69.00 & 18.5 & 1.084 \\
\hline $\begin{array}{l}4 . \\
5\end{array}$ & $\begin{array}{r}12 / 25 / 50 \\
2 / 18 / 50\end{array}$ & $\begin{array}{l}\begin{array}{l}3 / 15 \\
3 / 30\end{array} \\
\end{array}$ & $\begin{array}{l}161.0 \\
173.0\end{array}$ & $\begin{array}{l}46.00 \\
60.80\end{array}$ & $\begin{array}{l}13.0 \\
16.0\end{array}$ & $\begin{array}{l}1.073 \\
1.086\end{array}$ & $4 / 25$ & 172.0 & 55.70 & 13.5 & 1.073 & $6 / 03$ & 131.0 & 65.90 & 15.25 & 1.063 & $5 / 02$ & 183.2 & 73.90 & 16.75 & 1.056 \\
\hline 6. & $6 / 18 / 50$ & $4 / 05$ & 170.2 & 57.80 & 14.0 & $\begin{array}{l}1.086 \\
1.074\end{array}$ & $5 / 17$ & 175.0 & 62.00 & 17.5 & 1.076 & $5 / 04$ & 175.0 & 63.40 & 18.5 & 1.081 & $5 / 07$ & 175.5 & $\begin{array}{l}66.30 \\
79\end{array}$ & 19.0 & $\begin{array}{l}1.090 \\
1.083\end{array}$ \\
\hline 7. & $2 / 13 / 51$ & $4 / 26$ & 174.0 & 65.75 & 15.0 & $\begin{array}{l}1.074 \\
1.062\end{array}$ & $6 / 22$ & $\begin{array}{l}179.5 \\
180.0\end{array}$ & $\begin{array}{l}66.20 \\
72.90\end{array}$ & $\begin{array}{l}14.75 \\
15.5\end{array}$ & 1.075 & $6 / 21$ & 182.5 & $\begin{array}{l}75.50 \\
74.60\end{array}$ & 16.25 & 1.071 & $\begin{array}{l}11 / 18 \\
11 / 22\end{array}$ & $\begin{array}{l}183.0 \\
184.5\end{array}$ & $\begin{array}{l}79.30 \\
74.50\end{array}$ & 18.42 & $\begin{array}{l}1.083 \\
1.076\end{array}$ \\
\hline 8. & $9 / 14 / 50$ & $4 / 03$ & 183.0 & 78.16 & 16.5 & $\begin{array}{l}1.062 \\
1.081\end{array}$ & $\begin{array}{l}6 / 08 \\
6 / 09\end{array}$ & $\begin{array}{l}180.0 \\
186.5\end{array}$ & $\begin{array}{l}72.90 \\
82.40\end{array}$ & $\begin{array}{l}15.5 \\
18.0\end{array}$ & $\begin{array}{l}1.052 \\
1.091\end{array}$ & $\begin{array}{l}6 / 08 \\
6 / 21\end{array}$ & $\begin{array}{l}185.5 \\
187.0\end{array}$ & $\begin{array}{l}4.60 \\
87.70\end{array}$ & $\begin{array}{l}18.15 \\
18.5\end{array}$ & 1.080 & $6 / 20$ & 187.0 & 86.20 & 18.75 & 1.078 \\
\hline 9. & $7 / 10 / 50$ & $4 / 01$ & 160.5 & 47.00 & 14.0 & 1.058 & $5 / 26$ & 164.5 & 51.90 & 16.5 & 1.063 & $10 / 25$ & 166.0 & 56.40 & 18.0 & 1.075 & $4 / 26$ & 167.0 & 56.69 & 19.0 & 1.076 \\
\hline 10. & $5 / 09 / 50$ & $5 / 14$ & 170.0 & 66.30 & 15.15 & 1.059 & $6 / 13$ & 171.5 & 70.50 & 17.5 & 1.068 & $5 / 30$ & 172.8 & 69.80 & 18.25 & 1.071 & & 172.5 & 73.10 & 19.0 & 1.087 \\
\hline ii. & $5 / 11 / 50$ & $4 / 22$ & 171.0 & 66.10 & 14.75 & 1.045 & $5 / 31$ & 174.5 & 71.30 & 16.0 & 1.037 & $5 / 24$ & 176.8 & 70.50 & 17. & 1.0 & $4 / 30$ & 176.5 & 71.10 & 18.25 & \\
\hline 12. & $10 / 16 / 50$ & $4 / 13$ & 156.0 & 44.50 & 14.5 & 1.054 & $5 / 31$ & 164.0 & 50.40 & 13.75 & 1.070 & $4 / 20$ & 169.3 & 57.60 & 15 & 1.0 & $\begin{array}{l}5 / 02 \\
5 / 02\end{array}$ & 174.5 & 63.75 & 16.0 & 1.090 \\
\hline 13. & $1 / 13 / 50$ & $5 / 20$ & 183.0 & 60.20 & 16.0 & 1.080 & $5 / 27$ & 184.5 & 63.40 & 17.0 & 1.064 & $5 / 04$ & 185.0 & $\begin{array}{l}66.50 \\
550\end{array}$ & 18. & 1.073 & $5 / 24$ & 185.0 & 66.60 & 19.0 & \\
\hline 14. & $11 / 06 / 50$ & $6 / 04$ & 163.0 & 43.30 & 13.75 & 1.055 & $5 / 05$ & 170.5 & $\begin{array}{l}49.30 \\
64.20\end{array}$ & 12.75 & $\begin{array}{l}1.037 \\
1.056\end{array}$ & $5 / 11$ & 179.3 & $\begin{array}{l}59.60 \\
66\end{array}$ & 13.75 & 1.078 & $5 / 08$ & 182.5 & 64.20 & 16.5 & 1.081 \\
\hline 15. & $3 / 25 / 51$ & $4 / 08$ & 170.1 & 56.00 & 14.5 & 1.060 & $\begin{array}{l}5 / 27 \\
510\end{array}$ & $\begin{array}{l}178.0 \\
177.5\end{array}$ & $\begin{array}{l}64.20 \\
69.50\end{array}$ & 16.0 & $\begin{array}{l}1.056 \\
1.051\end{array}$ & $\begin{array}{l}5 / 15 \\
5 / 06\end{array}$ & $\begin{array}{l}180.0 \\
178.5\end{array}$ & $\begin{array}{l}67.30 \\
69.80\end{array}$ & 17.0 & 1.067 & $4 / 23$ & 182.0 & 69.40 & 17.75 & $\begin{array}{l}1.078 \\
1.078\end{array}$ \\
\hline 16. & $6 / 21 / 50$ & $\begin{array}{l}3 / 31 \\
3324\end{array}$ & $\begin{array}{l}174.5 \\
763.1\end{array}$ & $\begin{array}{l}61.80 \\
47.50\end{array}$ & $\begin{array}{l}16.0 \\
13.5\end{array}$ & $\begin{array}{l}1.076 \\
1.071\end{array}$ & $5 / 16$ & $\begin{array}{l}171.5 \\
172.0\end{array}$ & $\begin{array}{l}69.50 \\
54.00\end{array}$ & 14.75 & 1.059 & $\begin{array}{l}5 / 06 \\
5 / 04\end{array}$ & $\begin{array}{l}178.5 \\
175.0\end{array}$ & $\begin{array}{l}\begin{array}{l}9.80 \\
58.50\end{array}\end{array}$ & $\begin{array}{l}18.5 \\
16.5\end{array}$ & 1.068 & $\begin{array}{l}5 / 26 \\
4 / 25\end{array}$ & $\begin{array}{l}179.0 \\
176.8\end{array}$ & $\begin{array}{l}73.40 \\
60.50\end{array}$ & $\begin{array}{l}19.0 \\
17.5\end{array}$ & 1.085 \\
\hline 17. & $\begin{array}{r}8 / 20 / 50 \\
10 / 25 / 50\end{array}$ & $\begin{array}{l}3 / 24 \\
5 / 27\end{array}$ & $\begin{array}{l}15.1 \\
158.0\end{array}$ & $\begin{array}{l}4.50 \\
44.30\end{array}$ & 13.0 & 1.055 & $4 / 21$ & 165.5 & 51.10 & 13.75 & 1.092 & $5 / 15$ & 173.0 & 57.90 & $\begin{array}{l}16.5 \\
14.5\end{array}$ & $\begin{array}{l}1.071 \\
1.079\end{array}$ & $\begin{array}{l}44,25 \\
5 / 08\end{array}$ & 177.0 & $\begin{array}{l}61.50 \\
61.60\end{array}$ & 15.5 & 1.074 \\
\hline $\begin{array}{l}8.8 \\
19 .\end{array}$ & $11 / 18 / 50$ & $3 / 31$ & 166.2 & 49.00 & 14.0 & 1.073 & $5 / 03$ & 173.0 & 54.50 & 15.5 & 1.080 & $5 / 17$ & 175.3 & 59.40 & 17.0 & 1.074 & $5 / 13$ & 176.5 & 61.80 & 18.5 & 1.093 \\
\hline 20. & $6 / 21 / 50$ & $4 / 05$ & 168.3 & 53.40 & 14.5 & 1.046 & $5 / 11$ & 176.0 & 62.60 & 15.0 & 1.073 & $5 / 17$ & 180.0 & 67.80 & 16.5 & & $7 / 04$ & 181.5 & 72.90 & 18.25 & $\begin{array}{l}1.056 \\
1.066\end{array}$ \\
\hline 21. & $10 / 28 / 50$ & $5 / 18$ & 171.6 & 59.70 & 14.5 & 1.058 & $6 / 15$ & 177.0 & 61.50 & 16.25 & 1.0 & $5 / 19$ & 179.0 & 68.80 & 17.25 & 1.057 & $4 / 26$ & 179.5 & 70.40 & 18.25 & $\begin{array}{l}1.066 \\
1.071\end{array}$ \\
\hline 22. & $2 / 06 / 51$ & $4 / 21$ & 175.0 & 60.60 & 14.5 & 1.070 & $5 / 19$ & 180.0 & 68.90 & 16.75 & 1.049 & $4 / 26$ & 181.2 & 72. & 18.0 & 1.065 & $5 / 12$ & 183.0 & $\begin{array}{l}75.30 \\
78.77\end{array}$ & 18.75 & $\begin{array}{l}1.071 \\
1.083\end{array}$ \\
\hline 23. & $4 / 22 / 50$ & $3 / 15$ & 173.5 & 67.40 & 15.5 & 1.076 & $6 / 13$ & 177.5 & 72.50 & 17.0 & 1.074 & $4 / 26$ & 178.5 & 76. & 18. & 1.080 & $5 / 08$ & 179.0 & $\begin{array}{l}78.77 \\
65.00\end{array}$ & 19.0 & $\begin{array}{l}1.083 \\
1.084\end{array}$ \\
\hline 24. & $8 / 26 / 50$ & $5 / 31$ & 176.0 & 60.35 & 15.25 & 1.058 & $5 / 05$ & 178.0 & 65.00 & 16.75 & 1.0 & $5 / 22$ & 179.4 & 65. & 18.0 & 1.088 & $5 / 29$ & 179.0 & 66.00 & 18.75 & $\begin{array}{l}1.084 \\
1.068\end{array}$ \\
\hline 25. & $9 / 111 / 50$ & $5 / 03$ & 171.0 & 55.50 & 14.25 & 1.064 & $5 / 06$ & 175.0 & 61.30 & 15.75 & 1.051 & $5 / 29$ & 177.0 & 65.30 & 17.25 & 1.070 & $7 / 02$ & $\begin{array}{r}178.5 \\
69\end{array}$ & $\begin{array}{l}68.90 \\
62.50\end{array}$ & $\begin{array}{l}18.5 \\
18.0\end{array}$ & $\begin{array}{l}1.068 \\
1.076\end{array}$ \\
\hline 26. & $10 / 23 / 50$ & $3 / 17$ & 155.5 & 45.70 & 14.25 & 1.072 & $5 / 31$ & 164.0 & $\begin{array}{l}55.20 \\
56.10\end{array}$ & 15.0 & 1.064 & $\begin{array}{l}6 / 29 \\
4 / 21\end{array}$ & $\begin{array}{l}168.5 \\
176.5\end{array}$ & $\begin{array}{l}58.40 \\
59.10\end{array}$ & $\begin{array}{l}17.0 \\
18.0\end{array}$ & $\begin{array}{l}1.077 \\
1.076\end{array}$ & $\begin{array}{l}6 / 06 \\
5 / 13\end{array}$ & $\begin{array}{l}169.5 \\
177.0\end{array}$ & $\begin{array}{l}62.50 \\
62.30\end{array}$ & $\begin{array}{l}18.0 \\
18.75\end{array}$ & $\begin{array}{l}1.076 \\
1.082\end{array}$ \\
\hline 27. & $6 / 02 / 50$ & $\begin{array}{l}3 / 30 \\
5 / 12\end{array}$ & 170.2 & $\begin{array}{l}53.50 \\
57.40\end{array}$ & $\begin{array}{l}15.0 \\
15.0\end{array}$ & $\begin{array}{l}1.084 \\
1.074\end{array}$ & $\begin{array}{l}5 / 11 \\
5 / 06\end{array}$ & $\begin{array}{l}175.0 \\
175.5\end{array}$ & $\begin{array}{l}56.10 \\
61.30\end{array}$ & 17.0 & $\begin{array}{l}1.056 \\
1.073\end{array}$ & $\begin{array}{l}4 / 21 \\
5 / 23\end{array}$ & $\begin{array}{l}176.5 \\
176.5\end{array}$ & $\begin{array}{l}59.10 \\
73.80\end{array}$ & 18.0 & $\begin{array}{l}1.076 \\
1.058\end{array}$ & $\begin{array}{l}5 / 13 \\
5 / 03\end{array}$ & $\begin{array}{l}177.0 \\
178.3\end{array}$ & $\begin{array}{l}62.30 \\
66.50\end{array}$ & 18.15 & $\begin{array}{l}1.082 \\
1.074\end{array}$ \\
\hline 28. & $\begin{array}{r}8 / 09 / 50 \\
10 / 25 / 50\end{array}$ & $\begin{array}{l}5 / 12 \\
5 / 13\end{array}$ & $\begin{array}{l}173.2 \\
172.5\end{array}$ & $\begin{array}{l}57.40 \\
57.00\end{array}$ & $\begin{array}{l}15.0 \\
15.25\end{array}$ & $\begin{array}{l}1.074 \\
1.074\end{array}$ & $\begin{array}{l}5 / 00 \\
5 / 19\end{array}$ & 174.5 & 61.00 & 17.0 & 1.058 & $5 / 05$ & 175.0 & 64.10 & 17.75 & 1.085 & $6 / 03$ & 175.5 & 64.32 & 19.0 & 1.091 \\
\hline $\begin{array}{l}29 . \\
30\end{array}$ & $3 / 20 / 50$ & $5 / 13$ & 165.0 & 47.90 & 13.5 & 1.050 & $5 / 24$ & 172.5 & 55.60 & 15.0 & 1.051 & $6 / 05$ & 178.0 & 58.00 & 15.75 & 1.062 & $5 / 16$ & 179.1 & 61.30 & 16.5 & $\begin{array}{l}1.087 \\
1.053\end{array}$ \\
\hline $\begin{array}{l}30 . \\
31 .\end{array}$ & $1 / 21 / 50$ & $4 / 21$ & 168.0 & 68.70 & 15.25 & 1.035 & $5 / 11$ & 171.0 & 72.50 & 17.0 & 1.039 & $7 / 03$ & 171.0 & 68.60 & 18.25 & 1.054 & $4 / 26$ & 172.5 & 76. & & $\begin{array}{l}1.053 \\
1.076\end{array}$ \\
\hline 32. & $5 / 23 / 50$ & 4/22 & 175.0 & 54.40 & 14.0 & 1.058 & $5 / 06$ & 183.0 & 60.20 & 14.75 & 1.8 & $6 / 01$ & 186.3 & 66.30 & 17. & 1. & $11 / 20$ & 189.5 & 70. & & $\begin{array}{l}1.076 \\
1.091\end{array}$ \\
\hline 33. & $6 / 13 / 50$ & $4 / 74$ & 170.0 & 56.00 & 14.75 & 1.056 & $5 / 30$ & 179.5 & 64.00 & 15.5 & 1.054 & $6 / 08$ & 183.0 & 67.60 & 16. & 1.0 & $4 / 26$ & 184.5 & 70.00 & 18.0 & $\begin{array}{l}1.091 \\
1.074\end{array}$ \\
\hline 34. & $12 / 11 / 50$ & $4 / 12$ & 165.2 & 47.40 & 13.0 & 1.063 & $6 / 22$ & 176.0 & 56.30 & 13.75 & 1.075 & $6 / 20$ & 180.0 & 62.20 & 15 & 1.083 & $10 / 21$ & 182.5 & 64.40 & & $\begin{array}{l}1.074 \\
1.042\end{array}$ \\
\hline $\begin{array}{l}34 . \\
35 .\end{array}$ & $7 / 20 / 50$ & $5 / 06$ & 160.5 & 64.50 & 16.0 & 1.065 & $6 / 22$ & 162.0 & 67.00 & 18.25 & 1.077 & $5 / 04$ & 162.0 & 70.60 & 18. & 1.053 & $5 / 28$ & 163.0 & 76.40 & 19.0 & $\begin{array}{l}1.042 \\
1.064\end{array}$ \\
\hline 36. & $11 / 111 / 50$ & $4 / 14$ & 155.0 & 47.60 & 13.5 & 1.062 & $5 / 17$ & 163.5 & 54.90 & 14.75 & $1.05 \%$ & $5 / 18$ & 166.0 & 58.70 & 16.0 & 1.073 & $4 / 30$ & 168.0 & 61.50 & 16.75 & $\begin{array}{l}1.064 \\
1.044\end{array}$ \\
\hline 37. & $3 / 17 / 51$ & $3 / 30$ & 171.0 & 62.00 & 14.5 & 1.057 & $6 / 08$ & 173.5 & 69.70 & 16.5 & 1.043 & $6 / 21$ & 178.0 & 73.30 & 18.0 & 1.040 & $6 / 12$ & 178.0 & $\begin{array}{l}79.70 \\
65.50\end{array}$ & 18.5 & $\begin{array}{l}1.044 \\
1.072\end{array}$ \\
\hline 38. & $1 / 19 / 51$ & $3 / 31$ & 169.4 & 66.90 & 14.0 & 1.051 & $6 / 14$ & $\begin{array}{l}177.0 \\
185.0\end{array}$ & $\begin{array}{l}71.90 \\
65.30\end{array}$ & $\begin{array}{l}15.25 \\
15.75\end{array}$ & $\begin{array}{l}1.048 \\
1.082\end{array}$ & $\begin{array}{l}8 / 08 \\
4 / 27\end{array}$ & $\begin{array}{r}178.5 \\
187.0\end{array}$ & $\begin{array}{l}66.90 \\
68.30\end{array}$ & $\begin{array}{l}16.75 \\
17.0\end{array}$ & $\begin{array}{l}1.070 \\
1.075\end{array}$ & $\begin{array}{l}5 / 17 \\
5 / 14\end{array}$ & $\begin{array}{l}179.3 \\
187.5\end{array}$ & $\begin{array}{l}67.50 \\
67.32\end{array}$ & $\begin{array}{l}17.5 \\
18.25\end{array}$ & $\begin{array}{l}1.072 \\
1.085\end{array}$ \\
\hline 39. & $\begin{array}{l}4 / 21 / 50 \\
2 / 03 / 50\end{array}$ & $\begin{array}{l}5 / 04 \\
5 / 17\end{array}$ & $\begin{array}{l}181.5 \\
170.5\end{array}$ & $\begin{array}{l}59.75 \\
62.20\end{array}$ & $\begin{array}{l}14.5 \\
14.75\end{array}$ & $\begin{array}{l}1.087 \\
1.055\end{array}$ & $\begin{array}{l}5 / 10 \\
6 / 08\end{array}$ & $\begin{array}{l}185.0 \\
176.0\end{array}$ & $\begin{array}{l}61.90 \\
71.90\end{array}$ & $\begin{array}{l}15.75 \\
15.75\end{array}$ & 1.048 & $5 / 30$ & 179.2 & $\begin{array}{l}08.30 \\
71.10\end{array}$ & 17.75 & 1.072 & $7 / 08$ & 179.0 & 74.60 & 19.0 & 1.083 \\
\hline & & & & & & & & & & & & & & & & & & & & & \\
\hline
\end{tabular}

Alkaline phosphatase hypophosphatasia leuk ocyte

\title{
Hypophosphatasia: A Developmental Anomaly of Alkaline Phosphatase?
}

\author{
RAFAEL GORODISCHER, ${ }^{(28)}$ RONALD G. DAVIDSON, LUIS L. MOSOVICH, AND \\ SUMNER J. YAFFE \\ Department of Pediatrics, State University of New York at Buffalo, Buffalo, New York, USA
}

Extract

This report deals with quantitative and qualitative investigations of alkaline phosphatase in two unrelated infants with the severe infantile form of hypophosphatasia. Both affected infants had no detectable leukocyte alkaline phosphatase activities and both sets of parents and one sibling tended to have low but variable leukocyte enzyme activities. Normal duodenal juice alkaline phosphatase activity was present in the one patient in whom it was measured and a wide range of variation in enzymic activity was observed in the stools. There was no significant difference in the stool enzyme activity between both patients with hypophosphatasia $(42.01 \pm 9.77$ $\mathrm{U})$ and control infants $(40.55 \pm 6.29 \mathrm{U})$. However, the heterozygous parents had values significantly lower than the control adults $(2.10 \pm 0.47$ as compared with $19.10 \pm 4.44 \mathrm{U})$. Intestinal bacteria did not contribute significantly to the stool alkaline phosphatase activity. Enzyme activity was present in the bile of one of the patients and nearly absent in that of the other.
Three "inducers" of alkaline phosphatase were given to both patients (phenobarbital, vitamin $\mathrm{A}$, and corticosteroid). No clinical improvement or rise in serum alkaline phosphatase activity was observed during the trial of therapy with these agents. However, a significant increase in the activity of serum acid phosphatase was demonstrated during the course of vitamin A administration, suggesting an in vivo action of vitamin $\mathbf{A}$ on the lysosomes through decreasing the stability of the membrane and releasing acid phosphatase to the serum.

Quantitative determination of tissue alkaline phosphatases from autopsy tissues was highly variable: no activity was found in bone, lungs, or spleen of either infant; there was a discrepancy in liver and kidney alkaline phosphatase values (zero in one patient and present in the other) and activity was present in the intestinal mucosa of both.

Qualitative analysis of kidney, liver, and intestinal alkaline phosphatase revealed some differences between the patients and control subjects in heat inactivation and phenylalanine inhibition 